\title{
Erratum to: A Comparative Assessment of Observational Medical Outcomes Partnership and Mini-Sentinel Common Data Models and Analytics: Implications for Active Drug Safety Surveillance
}

Yihua $\mathrm{Xu}^{1} \cdot$ Xiaofeng Zhou $^{2} \cdot$ Brandon T. Suehs $^{1}$ (D) Abraham G. Hartzema ${ }^{3}$ • Michael G. Kahn ${ }^{4} \cdot$ Yola Moride $^{5}$ - Brian C. Sauer ${ }^{6}$ Q Qing Liu ${ }^{2} \cdot K_{\text {Keran Moll }}{ }^{1}$. Margaret K. Pasquale ${ }^{1} \cdot$ Vinit P. Nair $^{1} \cdot$ Andrew Bate $^{2}$

Published online: 2 July 2015

(c) Springer International Publishing Switzerland 2015

\section{Erratum to: Drug Saf (2015)}

DOI 10.1007/s40264-015-0297-5

An error was subsequently identified in the article, and the following correction should be noted:

Figures 2 and 3: The $X$-axis label in both figures was missing. The corrected figures should read:

The online version of the original article can be found under doi:10.1007/s40264-015-0297-5.

Brandon T. Suehs

bsuehs6@humana.com

1 Comprehensive Health Insights, Humana Inc., 515 W. Market St., Louisville, KY 40202, USA

2 Pfizer Inc., New York, NY, USA

3 College of Pharmacy, University of Florida, Gainesville, FL, USA

4 Department of Pediatrics, University of Colorado, Denver Anschultz Medical Campus, Aurora, CO, USA

5 Faculty of Pharmacy, Université de Montreal, Montreal, QC, Canada

6 Division of Epidemiology, Department of Internal Medicine, University of Utah, Salt Lake City, UT, USA 

Dimensional Propensity Score based analytic procedure results: fixed risk period (30 days starting from index date). $A M I$ acute myocardial infarction, $G I$ gastrointestinal, $O M O P$ Observational Medical
Fig. 2 Summary of High Outcomes Partnership

\begin{tabular}{|c|c|}
\hline Drug of Interest & Health Outcome \\
\hline Indomethacin & AMI \\
\hline Ketorolac & Gl Bleeding \\
\hline Benzodiazepine & Hip Fracture \\
\hline Valproic Acid & Acute Liver Injury \\
\hline Carbamazepine & Acute Liver Injury \\
\hline Amoxicillin & Anaphylactic Shock \\
\hline
\end{tabular}

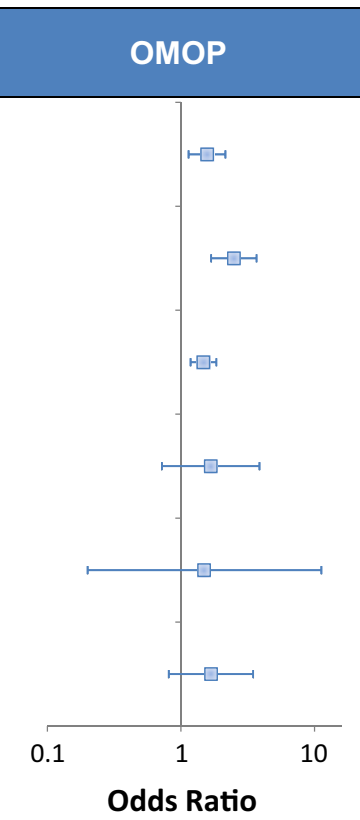

Mini-Sentinel

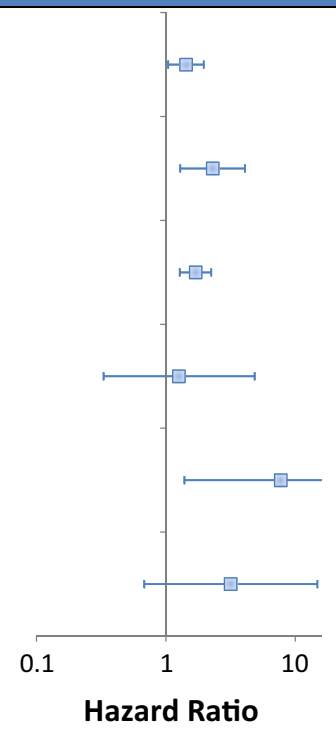

Fig. 3 Summary of High Dimensional Propensity Score based analytic procedure results: variable risk period (drug exposure time plus 30 days). $A M I$ acute myocardial infarction, GI gastrointestinal, $O M O P$ Observational Medical Outcomes Partnership
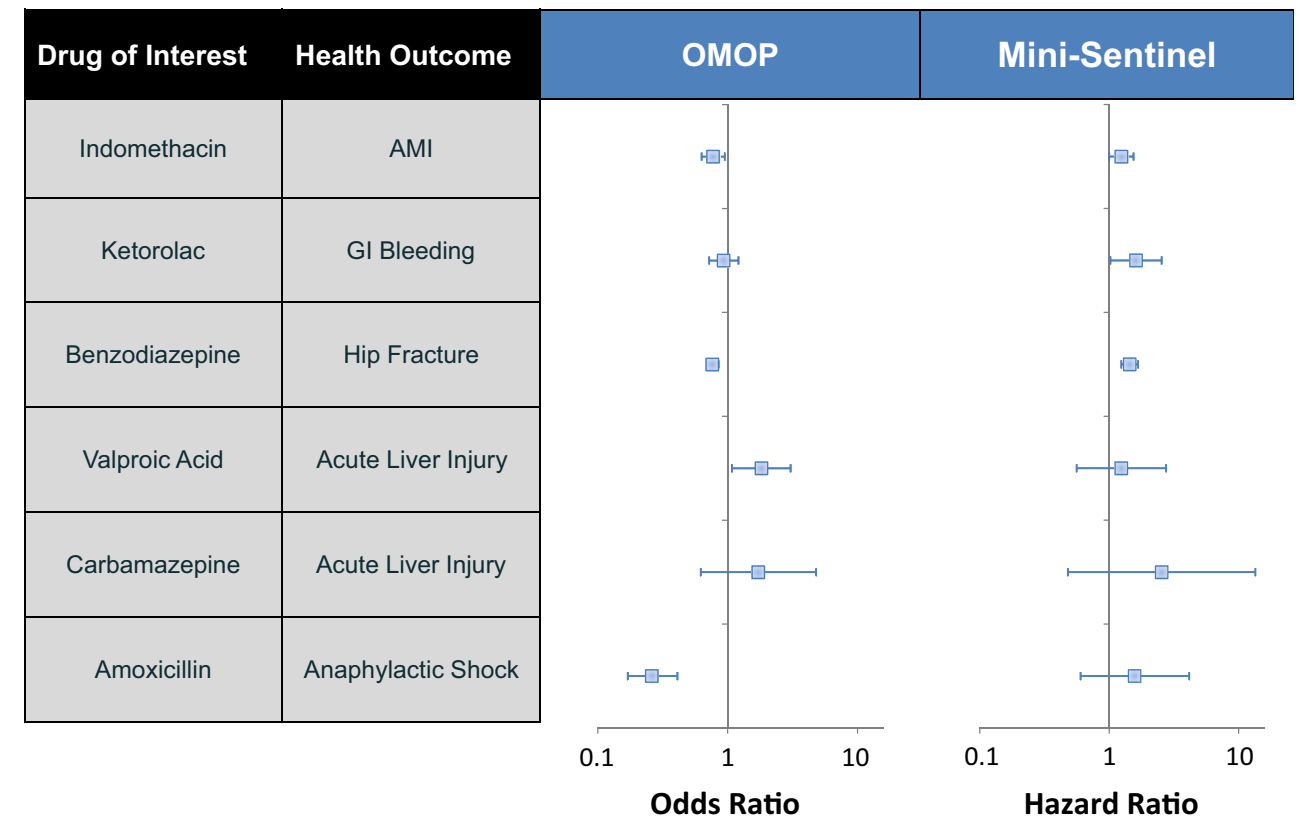